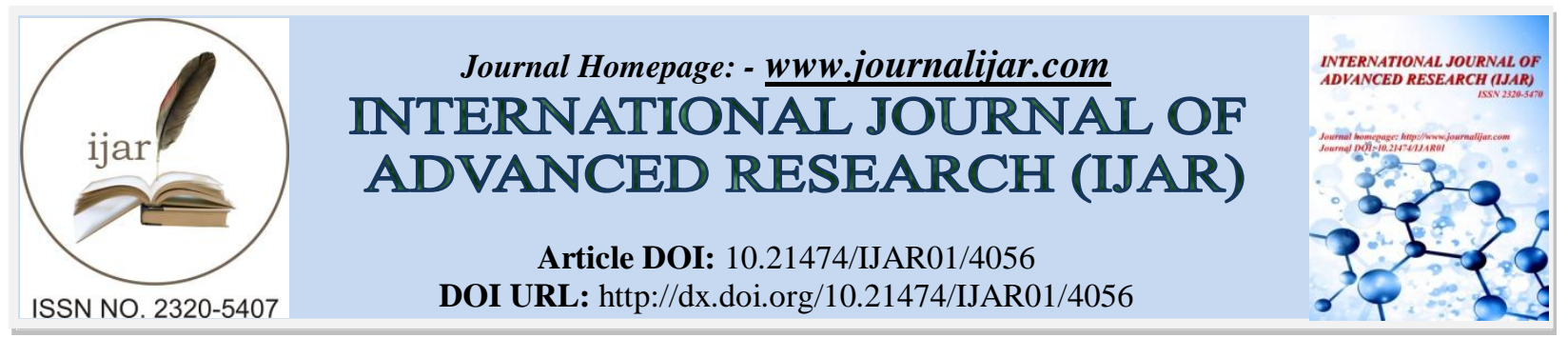

RESEARCH ARTICLE

\title{
LARGE JEJUNAL AV MALFORMATION WITH INTESTINAL ASCARIASIS PRESENTING AS OBSCURE GASTROINTESTINAL BLEED.
}

\author{
Jaswinder Singh Sodhi, Zaffer Ahmed Kawoosa, Sheikh Shaista, Mushqoor Ahmed, Mubasher Shah, Suresh \\ Gorka and Gulam Nabi Yattoo. \\ SKIMS Srinagar Kashmir,India.
}

\section{Manuscript Info \\ Manuscript History \\ Received: 21 February 2017 \\ Final Accepted: 26 March 2017 \\ Published: April 2017}

Key words:-

Obscure GI bleed, small bowel enteroscopy, , ascariasis Jejunal AVM

\begin{abstract}
Arterio venous malformations of small bowel constitute one of major causes of mid gastrointestinal bleed. We report a rare case of large Jejunal type 2 arterio venous malformations with intestinal ascariasis in a 59-year old male presenting as overt obscure gastro intestinal bleed with hemodynamic instability. Single balloon enteroscopy revealed jejunal ascariasis and a large serpignious vascular malformation involving whole of jejunum with a proximal bleeding spot, treated with argon plasma coagulation. In view of recurrent bleeding patient underwent exploratory laprotomy which revealed large arterio venous malformations involving whole jejunum along with acaroids. On enterotomy this vessel was opening into the mucosa with evidence of hemorrhage. Patient underwent resection anastomosis of the abnormal segment of jejunum and de wormed. Postoperative period was uneventful. Small bowel enteroscopy can be used as first line investigation in the diagnosis and management of suspected small bowel bleed. Type 2 large AV malformations invariable need surgical resection.
\end{abstract}

Copy Right, IJAR, 2017,. All rights reserved.

\section{Introduction:-}

Arterio venous malformations (AVM) of small bowel constitute one of major causes of mid gastrointestinal bleeding (MGIB) that is GI bleeding between the ampulla of Vater and the terminal ileum (Ell C, May A; 2006, Singh A, 2013) although small bowel bleed constitutes only 5-10 \% of GI bleed. (Zhang BL, 2012, Xin L, 2011). Capsule endoscopy (CE), balloon enteroscopy and CT enterography play a major role in the diagnosis of MGIB.(Sodhi etal,2013,2014) We report a rare case of large Jejunal type 2 AVM with intestinal ascariasis in a 59-year old male presenting as recurrent obscure overt GI bleed (OGIB) with hemodynamic instability. Single balloon enteroscopy (SBES) revealed a bunch of jejunal ascaroides and a large serpignious vascular malformation involving whole of jejunum with a proximal bleeding spot which was subjected to argon plasma coagulation (APC) with achievement of hemostasis. In view of recurrent bleeding exploratory laprotomy revealed almost whole of jejunum replaced with AVM and needed resection anastomosis of abnormal jejunal segment and deworming. Balloon enteroscopy can be used as first line investigation in cases of MGIB both for diagnostic and therapeutic utility. However endoscopic view of AVM may underestimate the actual size seen on laprotomy and eventually may require resection of the lesion. 


\section{Case Report:-}

A 59-year old male reported to Accident and Emergency department with complaint of passage of black colored stools multiple times at home with giddy spells. There was no history of abdominal pain, use of medications like NSAID, aspirin, and anticoagulants. Patient denied any history of trauma, jaundice, bleeding from any other site, rash, bloody stools, diarrhea, alteration in conscious levels, vomiting of blood or history of passage of worms. His physical examination revealed pulse of 120 beats/minute, Blood pressure on lying down 110/70 mm $\mathrm{Hg}$ with postural drop of Blood pressure greater than $10 \mathrm{mmHg}$ in sitting position. He had severe pallor. There was no jaundice or any stigmata of chronic liver disease. Systemic examination was normal. Ryles tube gastric aspirate was negative for blood. Lab. investigations revealed a hemoglobin $6.2 \mathrm{gm} / \mathrm{dl}$, total leukocyte count $8400 / \mathrm{cmm}$, Platelets $4.5 \mathrm{lacs} / \mathrm{cmm}$, Mean cell volume (MCV) $68 \mathrm{fl}$. Peripheral blood film revealed hypochromic microcytic cells with anisopoikilocytosis. Urea $62 \mathrm{mg} / \mathrm{dl}$, Creatinine $1.4 \mathrm{mg} / \mathrm{dl}$. Bilrubin $1.2 \mathrm{mg} / \mathrm{dl}$, AST $40 \mathrm{IU} / \mathrm{L}$, ALT $52 \mathrm{IU} / \mathrm{L}$, ALP 152 $\mathrm{IU} / \mathrm{L}$, Albumin $4.2 \mathrm{gm} / \mathrm{dl}$. Patient was resuscitated with two points of B positive blood transfusion.

Upper GI endoscopy revealed severe pallor of gastric mucosa. Ileo colonoscopy revealed malenic stools in left side of colon with fresh blood near cecum and ascending colon. Distal $15 \mathrm{~cm}$ of Ileum revealed blood stained mucosa without any lesion. In the hospital patient again reported passage of fresh blood along with stools with giddiness. His blood pressure was $90 \mathrm{mmHg}$ and was again resuscitated with two points of blood transfusion. Meckels scan was negative. In view of suspicion of MGIB patient was planned for antegrade (SBES). CT angiography could not be done in view of rise in serum creatinine.Patient could not afford capsule endoscopy in view of financial issues. Single balloon enteroscopy (SBES) was performed under general anesthesia which revealed large serpigneous vascular malformation starting from proximal jejunum up to proximal ileum. [Figure 1a, b,c]. There was evidence of hemorrhage and mild ooze of blood from the proximal end of vascular malformation. This area was subjected to APC which stopped the ooze. In addition to it there was a large bunch of ascaris seen in proximal jejunum (Figure 1d). During the next two days patient again had two episodes of hematochezia with fall in hemoglobin level.

In view of recurrent bleeding patient underwent exploratory laprotomy which revealed almost whole of serosal aspect of jejunum laden with extensive network of abnormal vessels arranged transversely and going towards mesenteric border. At places these vessels were tortuously dilated with thick hypertrophic walls and were running towards the mesentery laden with fat. One of the vessels was seen entering the wall of jejunum from mesentery and followed a long dilated serpignious course towards distal Jejunum [figure 2a, b, c,d]. On enterotomy this vessel was opening into the mucosa with evidence of hemorrhage. There were multiple worms seen in the segment of jejunum with AVM. Distal jejunum and ileum were normal. Patient underwent resection anastomosis of the abnormal segment of jejunum and dewormed. Postoperative period was uneventful. Patient has not reported any GI bleed since last 3 months follow-up. Biopsy of the resected jejunal segment revealed multiple, large cystic spaces in the sub mucosa, interspersed by loose connective tissue suggestive of jejunal AVM [figure 3a, b]. 

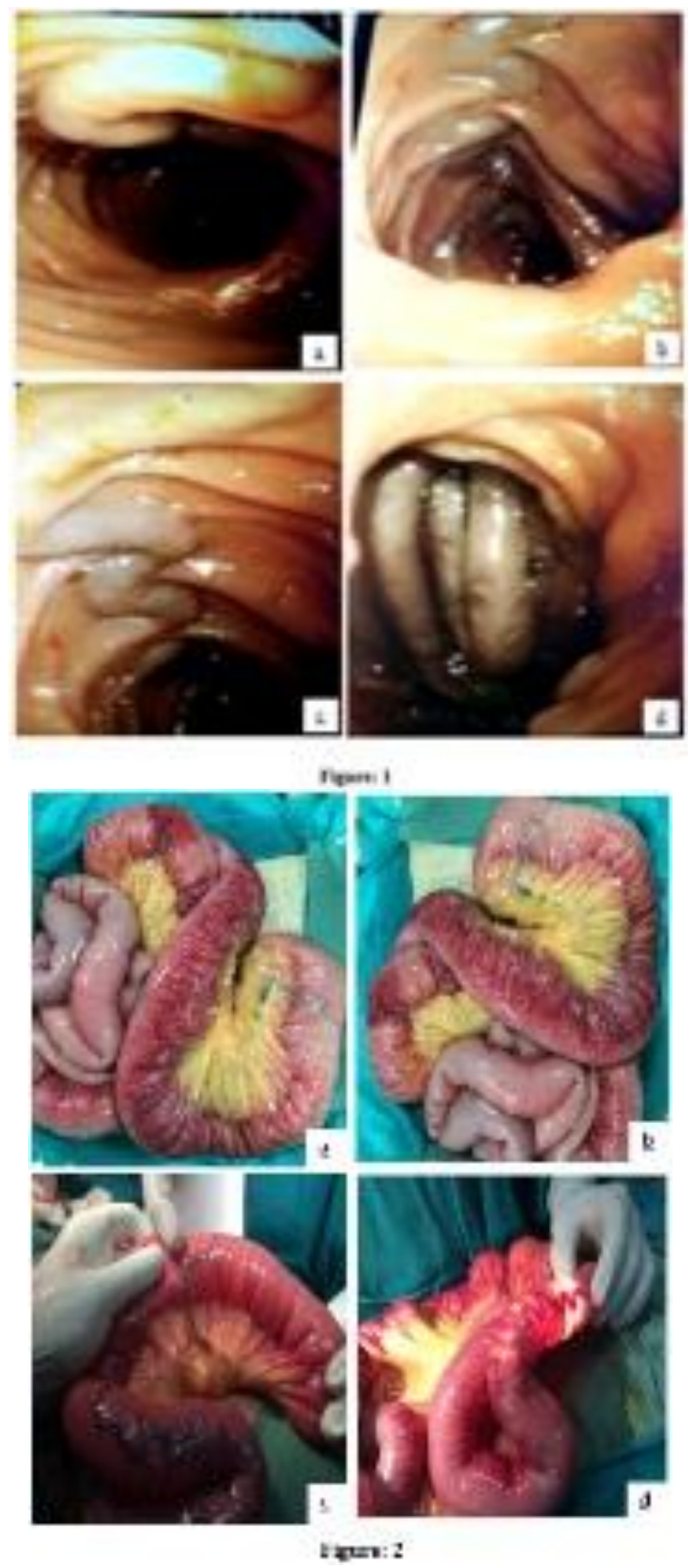

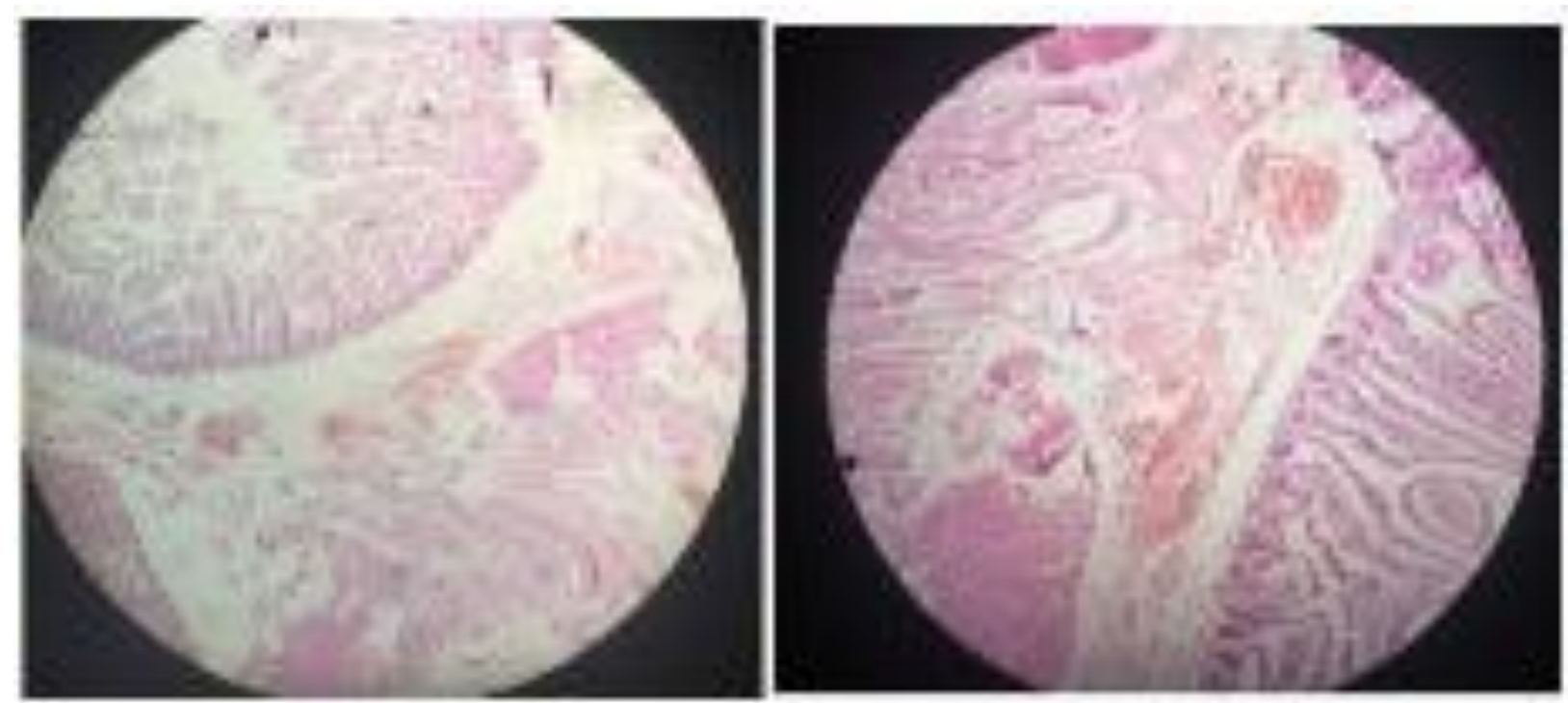

nres: 3

\section{Discussion:-}

This was a rare case of large type 2 Jejunal AVM with intestinal ascariasis who presented with major obscure overt GI bleed with hemodynamic instability. Although Intestinal ascariasis is endemic in Kashmir valley (India) however it is first time reported with a rare large type 2 jejunal AVM. The case was diagnosed by ante grade SBES. This case reveals the immediate therapeutic utility of SBE in arresting the hemorrhage by applying APC and also highlights that endoscopic picture of AVM may not correlate with actual size of AVM seen on laprotomy which was bigger compared to endoscopic picture. Although APC can arrest the bleeding immediately but in the long run bleeding invariably recurs and most of the time resection of AVM is required to prevent recurrent bleeding. (Jeffrey Kl,.2009, Endo H 2008, Fuji T, 2014) The case also may suggest that SBE can be a first line investigation for MGIB as it has both diagnostic and therapeutic utility.

Small bowel enteroscopy is used for the visualization of the small bowel by using single balloon enteroscope (SBE), double balloon enteroscope (DBE), and spiral enteroscope (SE). These endoscopes have both diagnostic and therapeutic utility and require an over tube for advancing the endoscope forward. Xin et al revealed a detection rate of 68\% for MGIB by using DBE which included detection of vascular lesions in (40.4\%), inflammatory (29.9\%), neoplastic (22.2\%), diverticulum (4.9\%) and others $(2.7 \%)$ of cases (Xin L,2011). The overall complications rates were $0.72 \%$, which included perforation, acute pancreatitis, bleeding and, aspiration pneumonia. Single balloon enteroscope has a single balloon at the tip of over tube and has similar diagnostic and therapeutic yield as in DBE. Single balloon enteroscopy is safe with low complication rate (Efthymiou M, 2012). The main pitfall of SBE is incomplete visualization of small bowel. The complete visualization is seen in only $11 \%$ of the patients using SBE compared 18\% in DBE (Domagk D, 2011).Capsule endoscopy (CE) enables the complete small bowel visualization non-invasively but it has no therapeutic utility. There is no difference in diagnostic yield of CE vs DBE (62\% vs $56 \%, \mathrm{p}=0.16)$ however, diagnostic yield of DBE improves to $75 \%$ if it was performed after a positive CE, whereas it was only $27.5 \%$ if the previously performed CE was negative (Jeffrey Kl, 2009, Teshima CW, 2011).

Small bowel constitutes 5\% of all GI bleeding. Arteriovenous malformations (AVM) cause 30 to $40 \%$ of MGIB. Arteriovenous malformations are an interconnection of aberrant arteries and veins and are classified as type 1 and 2 AVMS (Moore JD, 1976). Type 1 AVM or angiodysplasias (AD) histologically are thin-walled submucosal vessels that are dilated, often lined by endothelium only. They represent a degenerative process from partial or intermittent obstruction of sub mucosal veins, leading to capillary dilation, which results in arterio venous communication. Type 1 AVM or AD usually occurs in elderly patients and predominantly develops in the right colon (Moore JD, 2011, Thompson JN, 1984). 
Type 2 AVM is an interconnection of aberrant arteries and veins with thick hypertrophic walls. These lesions are usually congenital and are seen in young patients. Types 2 AVM are usually found in the stomach or jejunum. Although type I lesions can be shown with endoscopy or angiography, type II AVMs are not well seen by CT angiography because of their predominantly venous composition (Thompson JN ,1984). Type 2 AVMs in the small bowel can be demonstrated with enteroscopy or at surgery. Meyer et al. reviewed 218 cases of AVMs and found that the cecum or right colon was the most common location (78\%), whereas the jejunum (10.5\%), ileum (8.5\%) and duodenum (2.3\%) are other sites for AVM. (Meyer CT, 1981). Jejunal AVMs have been reported along with diverticula (Jeffrey Kl. 2009), and sometimes present as polypoid masses (Endo H,2008) and in our case it was associated with intestinal ascariasis.In our case the lesion seen on SBE was not correlating with the size of AVM on laprotomy which involved almost whole jejunum. In conclusion this was a rare case of large jejunal AVM associated with intestinal ascariasis.Small bowel enteroscopy may be the first choice in detection and also for arresting hemorrhage by using APC but invariably these patients require laprotomy because of recurrent bleed.

\section{References:-}

1. Ell C, May A. (2006): Mid-gastrointestinal bleeding: capsule endoscopy and push-andpull enteroscopy give rise to a new medical term. Endoscopy, 38:73-5.

2. Singh A, Baptista V, Stoicov C, et al. (2013): Evaluation of small bowel bleeding. Curr Opin Gastroenterol. 29:119-24.

3. Sodhi JS, Zargar SA, Wasim R, etal (2013) 64-section multiphase CTenterography as a diagnostic tool in the evaluation of obscure gastrointestinal bleeding,Indian J gastroenterol, 31(2):61-68

4. Sodhi JS, Ajaz A, Abid S, Khan BA, etal, (2013), Diagnostic role of capsule endoscopy in patients of obscure gastrointestinal bleeding after negative CT enterography ,journal digestive endoscopy, $4: 107-113$

5. Zhang BL, Chen CX, Li YM. (2012): Capsule endoscopy examination identifies different leading causes of obscure gastrointestinal bleeding in patients of different ages. Turk $\mathbf{J}$ Gastroenterol ; 23:220-5.

6. Xin L, Liao Z, Jiang YP, et al. (2011): Indications, detectability, positive findings, total enteroscopy, and complications of diagnostic double-balloon endoscopy: a systematic review of data over the first decade of use. Gastrointest Endosc. 74:563-70.

7. Jeffrey Kl. John MC, Pradipta G. (2009): ArterioVenous Malformation within Jejunal Diverticulum: An Unusual Cause of Massive Gastrointestinal Bleeding Gastroenterology Research and Practice DOI: $10.1155 / 2009 / 384506$.

8. Endo H, Matsuhashi N, Inamori M, etal (2008):Tumorous arteriovenous malformation in the jejunum missed by capsule endoscopy. Gastrointest Endosc; 68:773-774

9. Fujii T, Morita H, Sutoh T, Takada T, Tsutsumi S, Kuwano H. 92014): Arteriovenous Malformation Detected by Small Bowel Endoscopy Case Rep Gastroenterol; 8:324-328.

10. Efthymiou M, Desmond PV, Brown G, et al. (2012): SINGLE-01: a randomized, mcontrolled trial comparing the efficacy and depth of insertion of single- and double- balloon enteroscopy by using a novel method to determine insertion depth. Gastrointest Endosc; 76:972-80

11. Domagk D, Mensink P, Aktas H, et al. (2011): Single- vs. double-balloon enteroscopy in small bowel diagnostics: a randomized multicenter trial. Endoscopy; 43:472-6.

12. Teshima CW, Kuipers EJ, van Zanten SV, et al. (2011):Double balloon enteroscopy and capsule endoscopy for obscure gastrointestinal bleeding: an updated meta- analysis. JGastroenterol Hepatol; 26:796-801.

13. Moore JD, Thompson NM, Appleman HD, Foley D. (1976); Arteriovenous malformations of the gastrointestinal tract. Arch Surg ; 1 1:381-389

14. Thompson JN, Hemingway AP, McPherson GAD, Roes HC, Allison DJ Spencer J. (1984) Obscure gastrointestinal hemorrhage of small-bowel origin. BMJ ;288:1663-1665

15. Meyer CT, Troncale FJ, Galloway S, Sheahan DG. (1981):Arteriovenous malformations of the bowel; An analysis of 22 cases and a review of the literature. Medicine (Baltimore); 60; 36-48. 